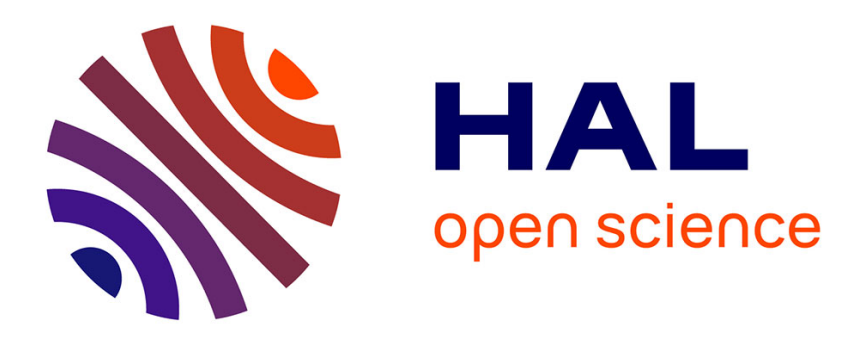

\title{
Étude cinétique par R. P. E. des atomes d'oxygène 3p dans la post-décharge
}

\author{
H. Tchen
}

\section{To cite this version:}

H. Tchen. Étude cinétique par R. P. E. des atomes d'oxygène 3p dans la post-décharge. Revue de Physique Appliquée, 1972, 7 (3), pp.205-212. 10.1051/rphysap:0197200703020500 . jpa-00243620

\section{HAL Id: jpa-00243620 https://hal.science/jpa-00243620}

Submitted on 1 Jan 1972

HAL is a multi-disciplinary open access archive for the deposit and dissemination of scientific research documents, whether they are published or not. The documents may come from teaching and research institutions in France or abroad, or from public or private research centers.
L'archive ouverte pluridisciplinaire HAL, est destinée au dépôt et à la diffusion de documents scientifiques de niveau recherche, publiés ou non, émanant des établissements d'enseignement et de recherche français ou étrangers, des laboratoires publics ou privés. 


\title{
ÉTUDE CINÉTIQUE PAR R. P. E. DES ATOMES D'OXYGËNE ${ }^{3} P$ DANS LA POST-DÉCHARGE
}

\author{
H. TGHEN \\ Laboratoire de Physique Moléculaire, \\ UER Structure et Propriétés de la Matière, Université de Rennes I
}

(Reçu le 7 février 1972, révisé le 24 mars 1972)

\begin{abstract}
Résumé. - Nous exposons la méthode du premier moment du signal d'absorption utilisée pour le calcul des concentrations en espèces paramagnétiques en phase gazeuse. Nous décrivons la réalisation et la mise au point de trois dispositifs expérimentaux de mesure et nous donnons les résultats obtenus dans l'étude par RPE de la recombinaison radicalaire dans la post-décharge d'un plasma froid d'oxygène à $2450 \mathrm{MHz}$.
\end{abstract}

Abstract. - The first moment method for the calculation of gas phase paramagnetic species is described. Three experimental arrangements to determine the absolute concentrations of atoms in a gas flow system at room temperature are described and results on the kinetic recombination rate constants in an oxygen afterglow are given.

I. Préliminaires. - La spectroscopie de Résonance du Spin Electronique permet de mesurer des concentrations absolues d'espèces paramagnétiques dans la phase gazeuse [1], [2], [3]. Dans le présent article, nous décrirons la réalisation et la mise au point d'un dispositif expérimental destiné à la mesure des concentrations d'espèces atomiques paramagnétiques formées dans une post-décharge. Le plasma est produit par excitation hyperfréquence d'un courant gazeux sous des pressions de l'ordre $\mathrm{du} \mathrm{mm} \mathrm{Hg}$.

II. Mesures des concentrations en espèces paramagnétiques. - 2.1 EXPRESSION DE LA CONCENTRATION. - Nous nous contenterons de rappeler brièvement la théorie que nous utilisons pour nos calculs ; pour de plus amples détails, le lecteur pourra se reporter à une publication antérieure [4]. L'intensité intégrée d'une raie d'absorption de résonance électronique est donnée par l'expression suivante :

$$
\begin{aligned}
\int_{-\infty}^{+\infty} \chi^{\prime \prime} \mathrm{d} H & =N \cdot \frac{\pi \omega_{0}}{2 \mu_{0} k T} \times \\
& \times g_{\alpha, J} \frac{\exp \left[-\varepsilon_{\alpha, J} / k T\right]}{\gamma_{\mathrm{e}} Z} \cdot\left|\left(\mu_{r}\right)_{J, M \rightarrow J^{\prime}, M^{\prime}}\right|^{2}
\end{aligned}
$$

$\chi^{\prime \prime}$ est la partie imaginaire de la susceptibilité paramagnétique de l'échantillon,

$N$ désigne le nombre de centres paramagnétiques par unité de volume d'échantillon,

$\omega_{0}$ la pulsation du champ hyperfréquence qui induit la transition entre les niveaux $\alpha, J, M$ et $\alpha^{\prime}$, $J^{\prime}, M^{\prime}$,

$\varepsilon_{\alpha, J}$ l'énergie du niveau $\alpha, J$ de dégénérescence $g_{\alpha, J}$, $\gamma_{\mathrm{e}}$ le rapport gyromagnétique effectif,

$Z$ - la fonction de partition pour un atome ou une molécule,

$\left|\left(\mu_{r}\right)_{J, M \rightarrow J^{\prime}, M^{\prime}}\right|^{2}$ le carré du module de l'élément de matrice de transition dans la direction du champ hyperfréquence.

Dans le cas d'une raie d'absorption correspondant à des transitions multiples partiellement résolues, l'expression précédente doit être sommée sur toutes les composantes de la raie :

$$
\begin{aligned}
& \int_{-\infty}^{+\infty} \chi^{\prime \prime} \mathrm{d} H=N \frac{\pi \omega_{0}}{2 \mu_{0} k T} \times \\
& \times \frac{\sum_{\alpha, J, M}\left[g_{\alpha, J} \exp \left(-\varepsilon_{\alpha, J} / k T\right)\left|\left(\mu_{r}\right)_{J, M \rightarrow J^{\prime}, M^{\prime}}\right|^{2}\right]}{\gamma_{\mathrm{e}} Z} .
\end{aligned}
$$

De l'expression ci-dessus, nous déduisons celle donnant le nombre $N$ de centres paramagnétiques par unité de volume:

$$
N=\frac{2 \mu_{0} k T}{\pi \omega_{0}} \cdot A \cdot \int_{-\infty}^{+\infty} \chi^{\prime \prime} \mathrm{d} H
$$

en posant :

$$
A=\frac{\gamma_{\mathrm{e}} Z}{\sum_{\alpha, J, M}\left[g_{\alpha, J} \exp \left(-\varepsilon_{\alpha, J} / k T\right)\left|\left(\mu_{r}\right)_{J, M \rightarrow J^{\prime}, M^{\prime}}\right|^{2}\right]} .
$$

Le facteur $A$ fait intervenir différents paramètres liés à la nature du gaz et à la transition considérée. L'évaluation de la concentration $N$ se ramène finalement au calcul de l'intensité intégrée. 
2.2 Méthode De Calcul [5]. - Il est possible de calculer l'intensité intégrée de trois manières :

a) Méthode de forme de raie valable seulement dans le cas des raies non déformées.

b) Méthode de double intégration du signal d'absorption.

c) Méthode du premier moment du signal d'absorption.

Pour notre part, nous avons choisi la méthode du premier moment du signal. Dans un précédent article [4], nous avons montré la validité de cette méthode et sa plus grande précision. Ce travail a été fait avec six raies du spectre RPE de l'oxygène moléculaire paramagnétique. Par rapport aux travaux de Westenberg et De Haas [6], nous avons apporté une contribution originale et conduisant à une correction théorique importante dans le calcul du premier moment. L'introduction de ce facteur correctif permet d'améliorer sensiblement la précision des calculs; ceci se traduit par l'obtention d'une précision de $4 \%$ sur le calcul des rapports des intensités intégrées de deux raies de $\mathrm{O}_{2}$ au lieu de $8 \%$ dans les travaux de ces auteurs.

Dans la méthode du premier moment du signal d'absorption, la concentration en espèces paramagnétiques est donnée par l'expression suivante [5]:

$$
N=C \cdot\left(H_{\mathrm{m}} H_{1} G\right)^{-1} A \cdot \lambda^{2} I
$$

avec :

$$
I=\int_{-\infty}^{+\infty}\left(x-x_{0}\right) S d x
$$

premier moment du signal

$$
\lambda=\frac{\mathrm{d} H}{\mathrm{~d} x} .
$$

$C$ est une constante liée à l'appareillage, $H_{\mathrm{m}}$ l'amplitude de modulation du signal, $H_{1}$ l'amplitude du champ hyperfréquence,

$G$ le gain du dispositif de réception du signal,

$\lambda=\mathrm{d} H / \mathrm{d} x$ dépend de la nature de l'échantillon étudié, et doit être mesuré pour chaque gaz,

$S$ est l'ordonnée de la dérivée première du signal d'absorption et $x$ son abscisse.

Dans la pratique, nous évaluons les concentrations absolues d'atomes par comparaison avec l'oxygène moléculaire. En opérant dans les mêmes conditions de modulation du signal et d'énergie hyperfréquence pour les deux gaz, la concentration atomique $N$ est égale à :

$$
N=N_{\mathrm{O}_{2}} \cdot \frac{A_{\mathrm{at}}}{A_{\mathrm{O}_{2}}} \cdot \frac{G_{\mathrm{O}_{2}}}{G_{\mathrm{at}}} \cdot\left(\frac{\lambda_{\mathrm{at}}}{\lambda_{\mathrm{O}_{2}}}\right)^{2} \cdot \frac{I_{\mathrm{at}}}{I_{\mathrm{O}_{2}}} .
$$

Nous utilisons cette dernière formule pour le calcul des concentrations atomiques à partir des enregistrements expérimentaux de la dérivée première de la courbe d'absorption. Le calcul numérique du premier moment $I$ est fait grâce à un programme à l'ordinateur Cï 10070 utilisant la méthode de Simpson [5].

III. Dispositif expérimental. - 3.1 DESCRIPTION GÉNÉRALE. - La figure 1 montre l'ensemble du dispositif expérimental utilisé qui comprend trois parties essentielles :

- le spectromètre de RPE Brüker de type Ber 402 b,

- le banc de mesure du champ magnétique continu,

- le dispositif de production du plasma.

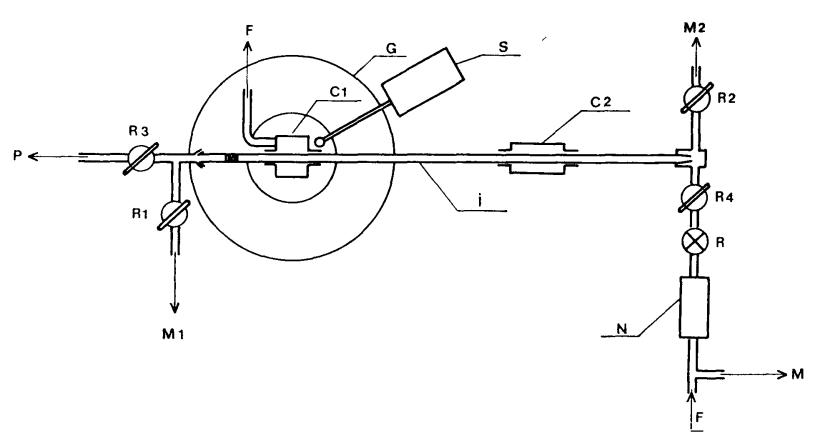

Fig. 1. - Ensemble du dispositif expérimental

$\mathrm{C}_{1}$ : Cavité RPE; $\mathrm{C}_{2}$ : Cavité à décharge ; E : Arrivée du gaz; $\mathrm{F}$ : Spectromètre RPE ; G : Electro-aimant ; I : Tube de silice ; $\mathrm{M}_{1} \mathrm{M}_{2}$ : Manomètres à membrane; $\mathrm{M}$ : Manomètre; $\mathrm{N}$ : Débitmètre; $\mathbf{P}$ : Pompes; $\mathrm{R}_{1} \mathbf{R}_{2} \mathrm{R}_{3} \mathrm{R}_{4}$ : Robinets à vide. $\mathrm{R}$ : Robinet à pointeau; $\mathrm{S}:$ Sonde protonique.

Le champ magnétique continu est mesuré au moyen d'un magnétomètre à résonance protonique $S$ réalisé au laboratoire et d'un fréquencemètre HewlettPackard [7], [8]. Pour chaque espèce paramagnétique étudiée, nous évaluons le facteur $\lambda=\mathrm{d} H / \mathrm{d} x$ pour différentes valeurs de la pression dans le domaine considéré pour nous assurer de sa constance.

L'écoulement gazeux est effectué à l'aide d'un système classique de pompes; les pressions amont et aval dans le tube de silice de qualité Suprasil sont mesurées avec deux manomètres à membrane $M_{1}$ et $\mathrm{M}_{2}$ qui donnent des indications absolues.

L'excitation du gaz est produite dans une cavité résonnante $C_{2}$ du type coaxial décrite par M. Peyron [9] et fonctionnant à $2450 \mathrm{MHz}$. Un banc de mesure hyperfréquence permet de connaître l'énergie incidente et l'énergie réfléchie par la cavité de décharge. L'énergie maximale disponible est d'environ $70 \mathrm{~W}$.

3.2 MÉThodes DE Mesure. - Nous avons utilisé trois méthodes expérimentales dans l'étude de la cinétique de recombinaison des atomes d'oxygène $\mathrm{O}\left({ }^{3} \mathrm{P}\right)$.

a) Méthode $A$ de variation de la pression. - C'est la première méthode que nous avons mise en œuvre [10]. Elle consiste à obtenir, la distance $C_{1} C_{2}$ étant maintenue constante, la variation du temps entre la création des atomes et leur détection en faisant varier la vitesse moyenne de l'écoulement gazeux, donc de la pression moyenne dans le tube de réaction. En effet, 
pour un flux laminaire, la vitesse moyenne de l'écoulement dans un tube de rayon $R$ et de longueur $L$ est donnée par:

$$
\bar{v}=\frac{\left(p_{1}-p_{2}\right) \cdot R^{2}}{8 v L}
$$

$p_{1}$ et $p_{2}$ étant les pressions à l'entrée et à la sortie du tube et $v$ la viscosité du gaz.

Cette méthode est laborieuse et nécessite un temps d'exploitation des résultats assez long, puisque chaque courbe de décroissance atomique comportant $k$ points nécessite le calcul de $2 k$ premiers moments du gaz étudié et de l'échantillon de référence. De plus, le nombre $k$ de points est limité par les possibilités de réglage des vannes amont et aval $\mathrm{R}_{3}$ et $\mathrm{R}_{4}(k$ de l'ordre de 8).

b) Méthode $B$ de déplacement de la source. - Lorsque, le régime des pressions étant stationnaire, on déplace la source atomique par rapport au système de détection ou vice-versa, le premier moment du signal d'absorption est proportionnel à l'amplitude pic à pic $S_{p p}$ de la dérivée première de ce signal (Fig. 2).

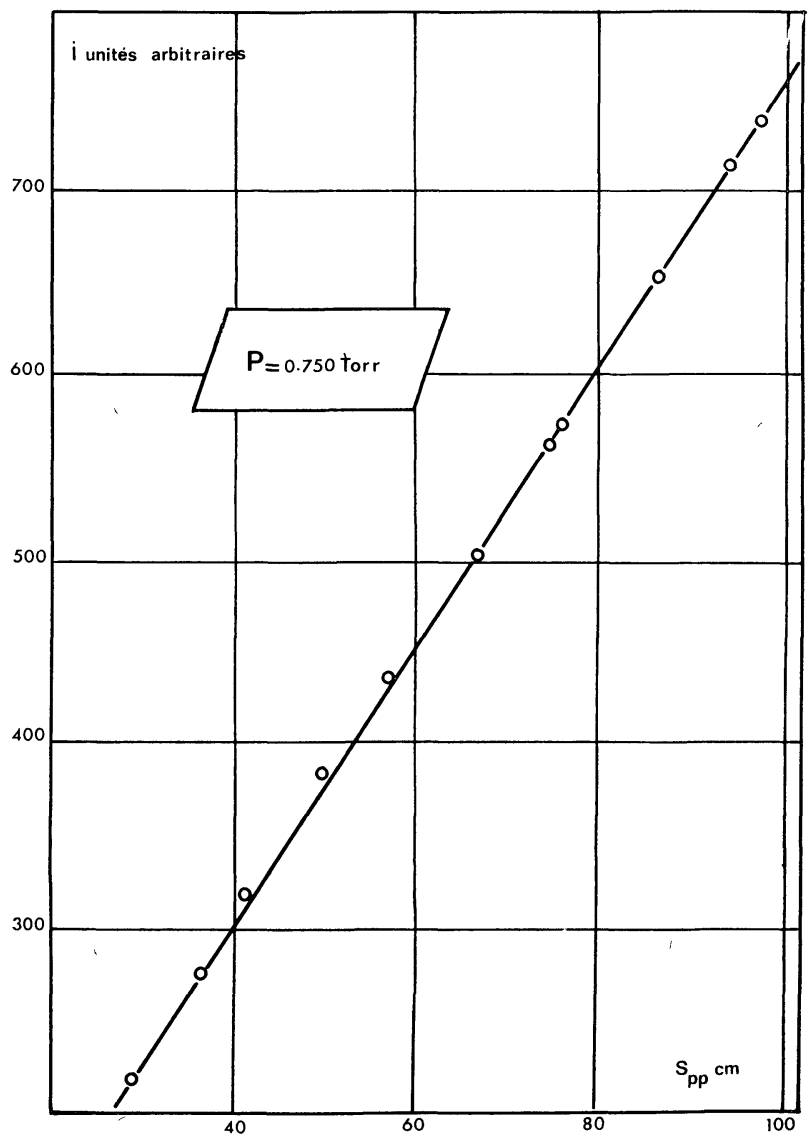

FIG. 2. - Courbe de variation du premier moment en fonction de l'amplitude pic à pic.

Il en résulte que l'enregistrement de deux signaux de RPE, l'un relatif au gaz étudié et l'autre à l'échantillon de référence suffit pour obtenir une courbe de décroissance atomique. Dans ce cas, on peut travail- ler sur un grand nombre d'abscisses et par conséquent obtenir un nombre $k$ de points supérieur au précédent ; ainsi pour nos expériences, sur une distance de $35 \mathrm{~cm}$ de déplacement total de la source, nous prenons quinze points et chaque point est la valeur moyenne d'au moins trois mesures d'amplitude pic à pic $\mathrm{S}_{\mathrm{pp}}$.

Avec cette méthode, le gain en temps est considérable. Le désavantage qu'elle peut avoir est l'existence d'un faible gradient de pression (environ $7 \%$ ) le long du tube de réaction ; ceci entraîne une incertitude sur le rendement atomique dans la décharge du gaz.

c) Méthode $C$ de déplacement du détecteur. Pour éliminer l'erreur introduite par l'existence du gradient de pression au niveau de la source atomique, il suffit de déplacer le système de détection, soit la cavité résonnante du spectromètre RPE et l'électro-aimant de douze pouces d'entrefer. Dans la pratique, pour éviter des difficultés techniques soulevées par le déplacement de l'électro-aimant, nous avons réalisé et mis au point le dispositif de la figure 3 moins onéreux et beaucoup plus pratique à manipuler.

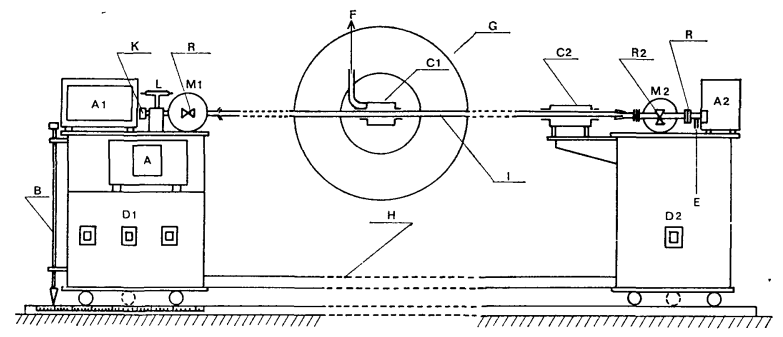

FIG. 3. - Système à translation.

$\mathrm{A}_{1} \mathrm{~A}_{2} \mathrm{~A}_{3}$ : Alimentations de jauges ; $\mathrm{B}$ : Repère de position ; $\mathrm{C}_{1}$ : Cavité RPE $; \mathrm{C}_{2}$ : Cavité à décharge $; \mathrm{D}_{1} \mathrm{D}_{2}$ : Chariots; $\mathrm{E}$ : Arrivée du gaz; $\mathrm{F}$ : Spectromètre RPE $; \mathrm{R}_{1} \mathrm{R}_{2}$ : Robinets à vide ; $\mathrm{G}$ : Electro-aimant ; $\mathrm{H}$ : Barre d'espacement ; $\mathrm{I}$ : Tube de silice ; $\mathrm{M}_{1} \mathrm{M}_{2}$ : Manomètres à membrane; $\mathrm{K}$ : Jauge à ionisation; L : Voyant lumineux ; R : Robinet à pointeau.

Dans cette version, nous déplaçons l'ensemble des pompes et appareils de contrôle de la pression, de la source atomique et du tube à écoulement par rapport à la cavité résonnante RPE par l'intermédiaire des deux chariots $D_{1}$ et $D_{2}$ rendus solidaires par une barre d'espacement $H$. Un repère de position $B$ placé devant une règle graduée donne à chaque instant la valeur de la distance source-détection. Enfin, pour empêcher toute vibration mécanique susceptible de se propager le long du bâti, la pompe primaire est reliée à la pompe secondaire au moyen d'un tuyau flexible en acier inoxydable d'une longueur de $1 \mathrm{~m}$.

Mais la mise au point de ce montage est rendue très délicate par le glissement du tube de silice dans la cavité RPE. Le problème le plus important est d'obtenir une sensibilité constante du système de détection tout le long du tube de réaction; pour satisfaire à cette condition très stricte :

- le centrage du tube de silice $(11 \mathrm{~mm}$ de dia- 
mètre extérieur) par rapport au passage dans la cavité résonnante du spectromètre RPE $(12 \mathrm{~mm}$ de diamètre) doit être parfait,

- sa flèche doit être la plus faible possible. Dans notre cas, elle est inférieure à $1 \mathrm{~mm}$ sur une longueur de $1 \mathrm{~m}$,

- et son homogénéité (épaisseur et pureté) doit être excellente.

Le contrôle de la sensibilité du système de détection est effectué, en l'absence d'excitation, par l'observation sur l'écran d'un oscillographe cathodique de la profondeur et de la position du pic d'absorption de la cavité résonnante. Après plusieurs tentatives et modifications, nous avons réussi à remplir cette condition, et ceci se traduit aussi par la disparition de points aberrants sur la courbe de décroissance atomique.

Cette troisième méthode de mesure bénéficie de tous les avantages de la précédente quant à l'exploitation des résultats expérimentaux.

IV. Résultats expérimentaux dans l'étude de la post-décharge de l'oxygène. - 4.1 CONDITIONS OPÉRATOIRES. - L'ensemble des expériences que nous avons effectuées avec les trois dispositifs décrits précédemment s'étend dans un domaine de pression de $0,5 \mathrm{~mm} \mathrm{Hg}$ à $2,0 \mathrm{~mm} \mathrm{Hg}$. Les vitesses moyennes d'écoulement gazeux sont calculées à partir de la formule (3.1) ou du débit. Avant chaque manipulation, le tube de silice est nettoyé au moyen d'un pompage secondaire prolongé. De plus, après avoir amorcé la décharge, nous la laissons se stabiliser pendant environ un quart d'heure pour obtenir un état de surface uniforme sur les parois du tube.

Les mesures de concentrations atomiques sont effectuées à partir d'une distance d'environ $30 \mathrm{~cm}$ en aval de la décharge. On élimine ainsi d'une part l'influence du' champ magnétique continu sur le plasma et d'autre part la présence d'électrons libres dans la post-décharge. De plus, en collaboration avec J. Burger, nous avons essayé de mesurer la densité électronique à la sortie de la cavité d'excitation au moyen d'un montage hyperfréquence mis au point au laboratoire [11]. A la limite de sensibilité de l'appareillage $\left(10^{10} \mathrm{~cm}^{-3}\right)$, nous n'avons pas pu mettre en évidence la présence d'électrons libres dans une région située à environ $4 \mathrm{~cm}$ de la décharge.

La raie de référence de l'oxygène moléculaire choisie comme référence (Fig. 4) est située à 5936 gauss ; elle correspond à la transition entre les composantes $M=1$ et $M=2$ du niveau $K=1, J=2$. Le spectre RPE de l'oxygène atomique (Fig. 5) est formé de deux raies dues aux transitions entre les niveaux $M_{J}$ dans le premier état excité ${ }^{3} \mathrm{P}_{1}$ et de quatre raies provenant des transitions entre les niveaux $M_{J}$ dans l'état fondamental ${ }^{3} \mathrm{P}_{2}$.

La figure 6 illustre trois courbes de décroissance atomique $a, b$ et $c$ obtenues respectivement avec les

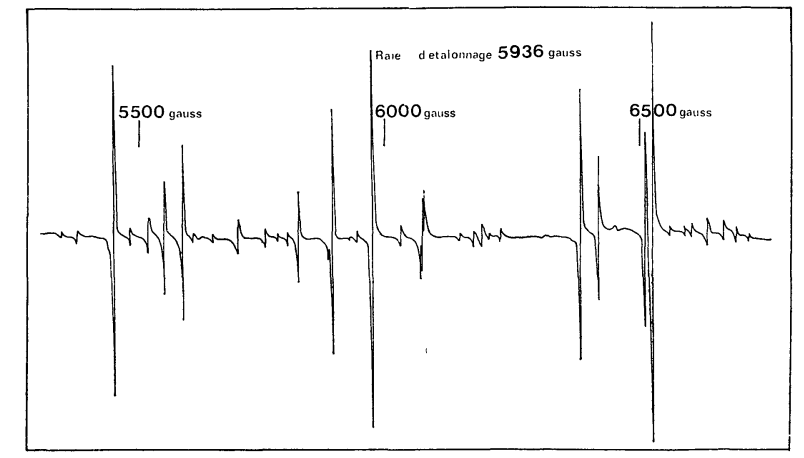

FIG. 4. - Spectre de RPE de l'oxygène moléculaire entre 5400 et 6700 gauss. Pression $=1,00$ torr.

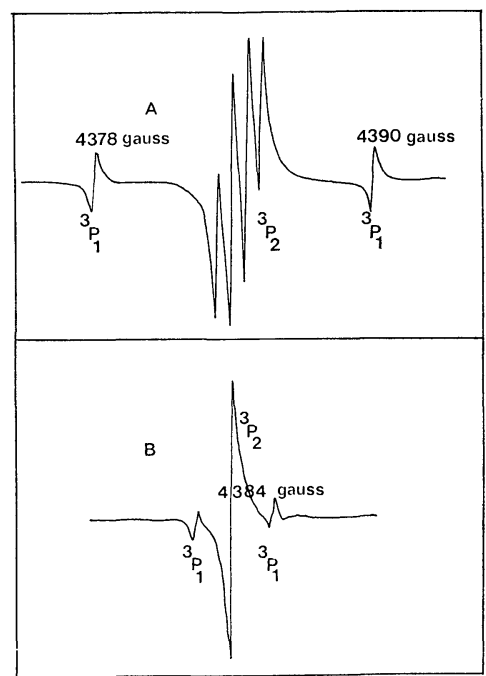

FIG. 5. - Spectre de RPE de l'oxygène atomique : A) $P=0,50$ torr ; B) $P=2,00$ torr.

trois méthodes de mesure $\mathrm{A}, \mathrm{B}$ et $\mathrm{C}$ à des valeurs de pression voisines. Les courbes sont tracées à partir d'un polynôme calculé par la méthode des moindres carrés à l'ordinateur CIII 10070 avec les valeurs expérimentales; nous avons constaté qu'après des essais préliminaires allant jusqu'au degré 5 , un polynôme de degré 3 suffit.

4.2 EquATION DE RECOMBINAISON. - Les processus de recombinaison intervenant dans un tel plasma sont les suivants :

I. Recombinaison à trois corps :

$\mathrm{O}+\mathrm{O}+\left[\mathrm{O}_{2}\right] \stackrel{k_{1}}{\rightarrow} \mathrm{O}_{2}+\left[\mathrm{O}_{2}\right]$ (en volume) .

II. Recombinaison en surface :

$$
\mathrm{O}+\mathrm{O}_{\text {adsorbé }} \stackrel{{ }^{-} k_{4}}{\rightarrow} \mathrm{O}_{2} \text {. }
$$

III. Mécanisme de l'ozone :

$$
\left.\begin{array}{c}
\mathrm{O}+\mathrm{O}_{2}+\left[\mathrm{O}_{2}\right] \stackrel{k_{5}}{\rightarrow} \mathrm{O}_{3}+\left[\mathrm{O}_{2}\right] \\
\mathrm{O}_{3}+\mathrm{O} \stackrel{k_{6}}{\rightarrow} 2 \mathrm{O}_{2}
\end{array}\right\} \text { (en volume). }
$$

IV. Recombinaison radiative :

$$
\mathrm{O}+\mathrm{O} \stackrel{k_{2}}{\longrightarrow} \mathrm{O}_{2}^{\text {r }}+h v \quad \text { (en volume) } \text {. }
$$




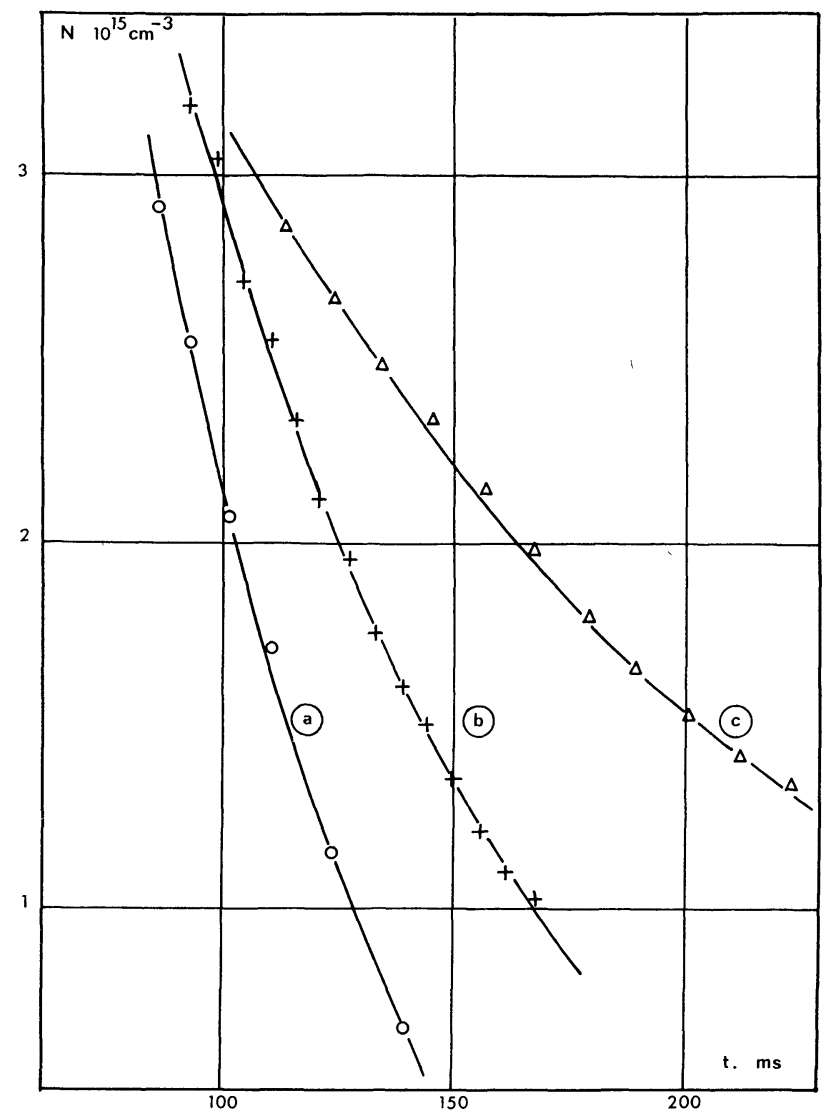

Fig. 6. - Courbes de décroissance atomique.

a) $P_{\text {déc }}=1,20$ torr ; b) $\bar{P}=1,52$ torr ; c) $\bar{P}=1,51$ torr.

Dans nos conditions expérimentales (pression de l'ordre du $\mathrm{mm} \mathrm{Hg}$ et faible rendement atomique), les mécanismes I et IV ont des contributions négligeables [12]. La variation au cours du temps de la concentration atomique $N$ dans une post-décharge à la température ambiante est alors régie par l'équation d'évolution déduite des équations de Navier-Stokes et de continuité [12] :

$$
-\frac{\mathrm{d}}{\mathrm{d} t} N=\left(2 k_{5} M^{2}+k_{4}\right) N-\frac{D}{\bar{v}^{2}} \frac{\mathrm{d}^{2}}{\mathrm{~d} t^{2}} N .
$$

$M$ désigne la concentration d'oxygène moléculaire en l'absence de décharge et $D$ le coefficient de diffusion atomique axiale. Le coefficient $k_{4}$ est relié à la probabilité de recombinaison en surface $\gamma$ par la formule :

$$
\gamma=\frac{2 R}{\bar{c}} \cdot k_{4}
$$

$\bar{c}$ est la vitesse thermique moyenne des atomes et $R$ le rayon du tube de réaction.

L'équation d'évolution (4.1) a été calculée en supposant une concentration constante d'ozone dans le mécanisme III ; ceci justifie la présence du facteur 2 dans le premier terme de l'équation.

4. 3 Constantes de Recombinaison. - La solu- tion de l'éq (4.1) qui décrit un processus du premier ordre obéit aux conditions limites :

$$
\begin{array}{ll}
\text { pour : } t=0 & N=N_{0} \\
\text { pour : } t=\infty & N=0 .
\end{array}
$$

Elle est de la forme :

$$
N=N_{0} \mathrm{e}^{-k^{\prime} t}
$$

avec :

$$
\begin{aligned}
& k=k^{\prime}\left(1+\frac{D}{\bar{v}^{2}} k^{\prime}\right) \\
& k=2 k_{5} M^{2}+k_{4} .
\end{aligned}
$$

$k^{\prime}$ est la pente de la courbe $g(t)=-\log N$.

En prenant pour le coefficient $D$ les valeurs données dans la littérature [12], [13], [14], nous avons calculé les valeurs maximales du terme $D / \bar{v}^{2} k^{\prime}$ pour chacune de nos expériences; elles n'excèdent jamais 0,1 . Par conséquent, le terme de diffusion axiale est négligeable.

-Finalement dans nos conditions expérimentales, les deux phénomènes les plus importants dans la post-décharge proviennent de la recombinaison au niveau des parois et du mécanisme de l'ozone. Nous avons tracé les courbes - $\log N=g(t)$ pour différentes valeurs de la pression à l'aide d'un programme à l'ordinateur CiII 10070 et nous avons constaté qu'elles sont en grande partie linéaires. La pente de ces courbes donne les valeurs de $\left(2 k_{5} M^{2}+k_{4}\right)$ pour les différentes valeurs de la pression, c'est-à-dire de

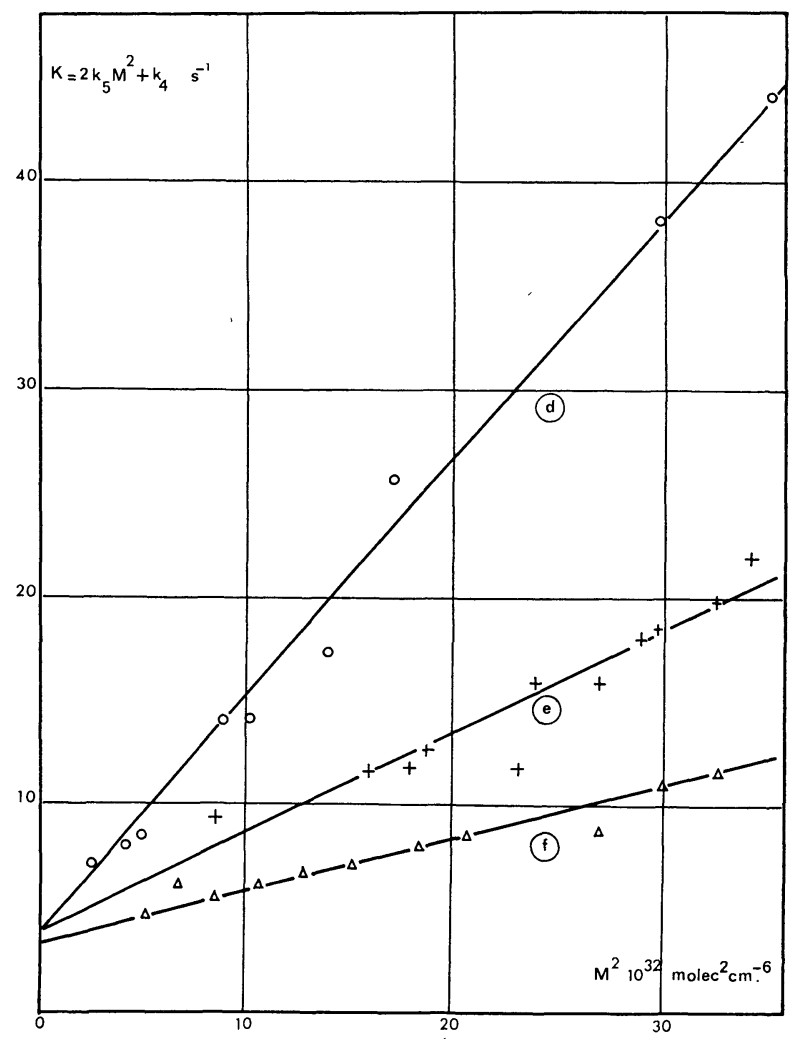

Fig. 7. - Courbes de variation de $K$ en fonction de $M^{2}$. 
TABLEAU I

Valeurs de $K=\left(\begin{array}{llll}2 & k_{5} & M^{2}+k_{4}\end{array}\right)$ en fonction de $M^{2}$

\begin{tabular}{|c|c|c|c|c|c|c|c|c|}
\hline \multicolumn{3}{|c|}{ Méthode A } & \multicolumn{3}{|c|}{ Méthode B } & \multicolumn{3}{|c|}{ Méthode C } \\
\hline $\begin{array}{c}P_{\mathrm{déc}} \\
\mathrm{mm} \mathrm{Hg}\end{array}$ & $\begin{array}{c}M^{2} \\
10^{32} \\
\mathrm{~cm}^{-6}\end{array}$ & $\begin{array}{c}\left(2 k_{5} M^{2}+k_{4}\right) \\
\mathrm{s}^{-1}\end{array}$ & $\begin{array}{c}P \\
\mathrm{~mm} \mathrm{Hg}\end{array}$ & $\begin{array}{c}M^{2} \\
10^{32} \\
\mathrm{~cm}^{-6}\end{array}$ & $\begin{array}{c}\left(2 k_{5} M^{2}+k_{4}\right) \\
\mathrm{s}^{-1}\end{array}$ & $\begin{array}{c}P \\
\mathrm{~mm} \mathrm{Hg}\end{array}$ & $\begin{array}{c}M^{2} \\
10^{32} \\
\mathrm{~cm}^{-6}\end{array}$ & $\begin{array}{c}\left(2 k_{5} M_{\mathrm{s}^{-1}}^{M^{2}}+k_{4}\right) \\
\end{array}$ \\
\hline 0,57 & 2,49 & 7,20 & 0,91 & 8,62 & 9,25 & 0,71 & 5,25 & 4,80 \\
\hline 0,73 & 4,30 & 8,00 & 1,25 & 16,09 & 11,50 & 0,81 & 6,83 & 6,16 \\
\hline 0,80 & 5,02 & 8,40 & 1,32 & 18,03 & 11,73 & 0,91 & 8,62 & 5,84 \\
\hline 0,89 & 9,12 & 14,00 & 1,35 & 18,78 & 12,65 & 1,01 & 10,62 & 6,40 \\
\hline 1,09 & 10,20 & 14,00 & 1,49 & 23,11 & 11,80 & 1,11 & 12,82 & 6,80 \\
\hline 1,29 & 13,90 & 17,20 & 1,52 & 23,89 & 15,87 & 1,21 & 15,23 & 6,92 \\
\hline 1,45 & 17,20 & 25,60 & 1,62 & 27,07 & 15,87 & 1,33 & 18,39 & 8,04 \\
\hline 1,90 & 29,68 & 38,00 & 1,67 & 29,01 & 17,94 & 1,41 & 20,67 & 8,64 \\
\hline 2,16 & 35,04 & 42,00 & 1,69 & 29,71 & 19,43 & 1,62 & 27,07 & 8,80 \\
\hline & & & 1,77 & 32,55 & 19,78 & 1,70 & 30,03 & 11,15 \\
\hline & & & 1,81 & 34,07 & 21,96 & 1,77 & 32,55 & 11,61 \\
\hline
\end{tabular}

la concentration moléculaire $M$. Les valeurs des constantes $2 k_{5}$ et $k_{4}$ sont ensuite déterminées à partir de la droite de variation de $K=\left(2 k_{5} M^{2}+k_{4}\right)$ en fonction de $M^{2}$.

La figure 7 montre les trois droites $d$, e et $f$ que nous avons obtenues avec les trois méthodes de mesure décrites précédemment. Chaque point correspond à une courbe de décroissance atomique pour une valeur donnée de la pression. Dans le tableau I, pour la méthode $\mathrm{A}$, la pression $P_{\text {déc }}$ est la valeur de la pression au niveau de la décharge, tandis que pour les deux autres méthodes, $P$ est la valeur de la pression moyenne dans le tube de réaction.

Les valeurs des deux coefficients de recombinaison $k_{4}$ et $k_{5}$ déduits de ces trois courbes sont consignées dans le tableau suivant :

\section{TABLEAU II}

Valeurs des coefficients de recombinaison dans la post-décharge de l'oxygène

\begin{tabular}{cllll} 
Méthode de mesure & \multicolumn{1}{c}{ A } & B & C \\
$k_{4} \mathrm{~s}^{-1} \ldots \ldots \ldots \ldots \ldots \ldots \ldots \ldots$ & 4 & - & $-2,3$ & 3,8 \\
$k_{5} \times 10^{-33} \mathrm{~cm}^{6}$ mol. $^{-2} \mathrm{~s}^{-1} \ldots$ & 5,5 & 2,7 & 1,2
\end{tabular}

Dans les expériences faites avec la méthode $\mathrm{A}$, nous avons utilisé les valeurs des coefficients $k_{4}$ et $k_{5}$ pour déterminer le coefficient $k_{1}$ dans la partie non linéaire des courbes $g(t)=-\log . N$. Nous avons obtenu une valeur $k_{1}=10^{-32} \mathrm{~cm}^{6}$ mol..$^{-2} \mathrm{~s}^{-1}$ en résolvant numériquement l'équation d'évolution de la densité atomique qui décrit l'ensemble des trois processus de recombinaison I, II et III :

$-\frac{\mathrm{d}}{\mathrm{d} t} N=\left(2 k_{5} M^{2}+k_{4}\right) N+k_{1} M N^{2}-\frac{D}{\bar{v}^{2}} \frac{\mathrm{d}^{2}}{\mathrm{~d} t^{2}} N$.
L'examen du tableau II montre que les valeurs obtenues pour le coefficient de recombinaison en surface $k_{4}$ par les trois méthodes $\mathrm{A}, \mathrm{B}$ et $\mathrm{C}$ sont du même ordre de grandeur. Quant au coefficient de recombinaison en volume $k_{5}$, l'écart entre les valeurs obtenues est plus grand. La valeur élevée obtenue par la méthode A est due d'une part, aux sources d'erreur liées à la stabilité des pressions et des vitesses d'écoulement et à leurs mesures, et d'autre part, au nombre beaucoup trop limité de points expérimentaux pour décrire la courbe de décroissance atomique. Bien qu'elle soit délicate à mettre en œuvre, la méthode $\mathrm{C}$ conduit certainement aux meilleurs résultats puisqu'elle comporte le minimum de sources d'erreur.

Dans le tableau III, nous avons consigné les valeurs des trois coefficients de recombinaison $k_{1}, k_{4}$ et $k_{5}$ que nous avons pu trouver dans la littérature. Pour la recombinaison en surface, la comparaison est effectuée avec le coefficient de probabilité $\gamma$ déduit de la formule (4.2) ; l'accord entre nos résultats et ceux des autres auteurs utilisant une technique identique ou différente est très satisfaisant et indique le rôle important de ce processus de recombinaison dans les décharges fonctionnant sous des pressions de l'ordre du $\mathrm{mm} \mathrm{Hg}$ ou inférieures.

Pour le coefficient de recombinaison $k_{5}$, nos valeurs sont en bon accord avec celles de Dickens [16] et Harteck [17], elles sont légèrement supérieures à celles des autres chercheurs. Cette différence peut s'expliquer par le fait que ces derniers ne font intervenir dans leurs études qu'un seul processus de recombinaison. Par ailleurs, nous trouvons des résultats en excellent accord avec ceux des chercheurs qui ont essayé d'inclure plusieurs phénomènes. Le problème qui peut se poser dans notre cas est celui de la présence d'atomes d'oxygène dans des états excités autres que l'état ${ }^{3} \mathrm{P}$. Cependant, la présence du premier état excité ${ }^{1} \mathrm{D}$ le plus probable n'a pas pu être mise en 


\section{TABLEAU III}

Valeurs des coefficients de recombinaison dans un plasma d'oxygène à la température ambiante

\begin{tabular}{|c|c|c|c|c|c|c|}
\hline Auteurs & $\begin{array}{c}\text { Technique } \\
\text { Expérimentale }\end{array}$ & $\begin{array}{c}\text { Nature } \\
\text { des parois }\end{array}$ & $\begin{array}{cc}k_{1} \quad 10^{-32} \\
\mathrm{~cm}^{6} \mathrm{~mol}^{-2} \mathrm{~s}^{-1}\end{array}$ & $\begin{array}{c}k_{5} 10^{-33} \\
\mathrm{~cm}^{6} \mathrm{~mol}^{-2} \mathrm{~s}^{-1} \\
-\end{array}$ & $\begin{array}{l}k_{4} \\
\mathrm{~s}^{-1} \\
-\end{array}$ & $\begin{array}{c}\gamma \\
10^{-4} \\
-\end{array}$ \\
\hline Greaves, Linnett [15] & $\begin{array}{l}\text { Ecoulement, décharge } \mathrm{O}_{2} \\
\text { Jauge de Wredge }\end{array}$ & Silice & & & & 1,6 \\
\hline $\begin{array}{l}\text { Krongelb, Strandberg } \\
\text { [2] }\end{array}$ & $\begin{array}{l}\text { Ecoulement, décharge } \mathrm{O}_{2}, \\
\text { RPE }\end{array}$ & Silice & 1,4 & & 2,0 & 3,2 \\
\hline Dickens \& coll. [16] & Ecoulement, décharge $\mathrm{O}_{2}$ & Pyrex & & 1,28 & 1,9 & 1,2 \\
\hline & Sonde thermique & & & $1,67^{*}$ & 1,7 & 1,1 \\
\hline Harteck \& coll. [17] & $\begin{array}{l}\text { Ecoulement, décharge } \mathrm{O}_{2} \\
\text { Post-luminescence }\end{array}$ & & & 2,70 & & \\
\hline Hacker \& coll. [18] & $\begin{array}{l}\text { Ecoulement, décharge } \mathrm{O}_{2} \\
\text { RPE }\end{array}$ & Silice & & & & \\
\hline Marshall [19] & $\begin{array}{l}\text { Ecoulement, décharge } \mathrm{O}_{2} \\
\text { RPE }\end{array}$ & Pyrex & 0,45 & & 7,9 & 1,7 \\
\hline Benson, Axworthy [20] & $\begin{array}{l}\text { Décomposition thermique } \\
\text { de } \mathrm{O}_{3}\end{array}$ & & & 0,36 & & \\
\hline Wray [21] & $\begin{array}{l}\text { Tube à ondes de choc } \mathrm{O}_{2} \\
\text { Spectroscopie d'absorption }\end{array}$ & & & $29,5 * *$ & & \\
\hline $\begin{array}{l}\text { Williams, Mulcahy } \\
\text { [22] }\end{array}$ & $\begin{array}{l}\text { Pyrolyse } \mathrm{O}_{3} \\
\text { Chimiluminescence }\end{array}$ & Pyrex & & 0,88 & $4 \ll 8$ & \\
\hline Kaufman, Kelso [23] & $\begin{array}{l}\text { Pyrolyse } \mathrm{O}_{3} \\
\text { Chimiluminescence }\end{array}$ & & & 0,65 & & \\
\hline Francis [24] & $\begin{array}{l}\text { Ecoulement, décharge } \mathrm{O}_{2} \\
\text { RPE }\end{array}$ & & & & & \\
\hline Slanger, Black [25] & $\begin{array}{l}\text { Pyrolyse } \mathrm{O}_{2} \\
\text { Fluorescence de résonance }\end{array}$ & & & 0,72 & & \\
\hline Stuhl, Niki [26] & $\begin{array}{l}\text { Photolyse U. V. } \mathrm{O}_{2} \\
\text { Chimiluminescence }\end{array}$ & & & 0,64 & & \\
\hline $\begin{array}{l}\text { Nos résultats anté- } \\
\text { rieurs [10] }\end{array}$ & $\begin{array}{l}\text { Ecoulement, décharge } \mathrm{O}_{2} \\
\text { RPE }\end{array}$ & Silice & $\sim 1$ & 5,5 & 4 & 1,4 \\
\hline Nos résultats actuels & $\begin{array}{l}\text { Ecoulement, décharge } \mathrm{O}_{2} \\
\text { RPE }\end{array}$ & Silice & & $\begin{array}{l}2,7 \\
1,2\end{array}$ & $\begin{array}{l}2,3 \\
3,8\end{array}$ & $\begin{array}{l}0,8 \\
0,95\end{array}$ \\
\hline
\end{tabular}

(*) Valeur obtenue en tenant compte de la diffusion atomique.

$(* *)$ Valeur calculée par extrapolation des résultats obtenus à $5000^{\circ} \mathrm{K}$.

évidence par Marshall [19] ; ceci peut s'interpréter par une déactivation collisionnelle rapide de cet état suivant la réaction :

$$
\mathrm{O}\left({ }^{1} \mathrm{D}\right)+\mathrm{O}_{2} \rightarrow \mathrm{O}\left({ }^{3} \mathrm{P}\right)+\mathrm{O}_{2}\left({ }^{1} \Sigma_{\mathrm{g}}^{+}\right)+\mathrm{O}_{2}\left({ }^{1} \Delta_{\mathrm{g}}\right) .
$$

Enfin la valeur du coefficient $k_{1}$ que nous avons déterminée est en bon accord avec celles de Krongelb [2] et Marshall [19]. Un calcul théorique de ce coefficient a été effectué par Bauer [27]. En admettant que le processus de recombinaison a lieu dans les états excités vibrationnels élevés $(v=9,10)$ du niveau ${ }^{3} \Sigma_{u}^{+}$de l'oxygène moléculaire, il trouve une valeur comprise entre $1 \times 10^{-33}$ et $10 \times 10^{-33} \mathrm{~cm}^{6} \mathrm{moe}^{-2} \mathrm{~s}^{-1}$.
V. Conclusion. - Nous avons décrit trois méthodes de mesure par RPE des coefficients de recombinaison dans la post-décharge d'un plasma d'oxygène sous des pressions de l'ordre du $\mathrm{mm} \mathrm{Hg}$. Nos résultats sont d'une façon générale en bon accord avec ceux cités dans la littérature. Dans un prochain stade, il sera intéressant d'entreprendre l'étude soit de l'influence de différents paramètres sur la recombinaison au niveau des parois (nature, température des parois), soit de l'efficacité de recombinaison du troisième corps dans le processus de recombinaison à trois corps. Ce dernier phénomène offre un intérêt certain dans l'étude de la haute atmosphère et de la pollution de l'environnement par les gaz de combustion.

\section{Bibliographie}

[1] Mc. Taggart (F. K.), « Plasma Chemistry in Electrical Discharges ", Elsevier Pub. Co., 1967.

[2] Krongelb (S.), Strandberg (M. W. P.), J. Chem. Phys., 1959, 31, 5, 1196.

[3] Kaufman (A.), Adv. Chem., 1969, Ser. 80, 29.

[4] Herry (J. Y.), Marchand (A.), Mlle Raphalen (M. N.), TCHEN (H.), GAMS 1970, $\mathrm{n}^{\circ} 1,60$.

[5] Herry (J. Y.), Thèse 3e cycle Rennes, 1969.
[6] Westenberg (A. A.), De HaAs (N.), J. Chem. Phys, 1964, 40, 3087

[7] TChen (H.), Thèse $3^{\mathrm{e}}$ cycle Rennes, 1965.

[8] Tchen (H.), Marchand (A.), Bull. Soc. Sci. Bretagne, 1967, 42, 53.

[9] Peyron (M.), J. Chim. Phys., 1962, 59, 1.

[10] Mlle Raphalën (M. N.), Tchen (H.), C. 'R. Acad. Sci., Paris, 1970, 271, 21. 
[11] Burger (J.), Thèse $3^{\mathrm{e}}$ cycle Rennes, 1968.

[12] Mlle RAphalËN (M. N.), Thèse 3e cycle Rennes, 1970.

[13] Kaufman (F.), Progr. React. Kin., 1961, $1,1$.

[14] Andrussov (L.), Z. Electrochem., 1950, 54, 566.

[15] Greaves (J. C.), Linnett (J. W.), Trans. Farad. Soc., 1959, 55, 1338.

[16] Dickens (P. G.), Gould (R. D.), Linnett (J. W.), RichMOND (A.), Nature, 1960, 187.

[17] Harteck (P.), Reeves (R. R.), Manella (G.), AFSOR. TR., 57-50, no AD., 136.421, 1960.

[18] Hacker (D. S.), Marshall (S.), Steinberg (M.), - VIIIth Inter. Symp. on Combustion, 1960.

[19] Marshall (T. C.), Phys. Fluids, 1962, 5, 7, 743.
[20] Benson (S. W.), Axworthy (A. E.), J. Chem. Phys., 1965, 42, 2614.

[21] Wray (K. L.), Xth Intern. Symp. on Combustion 1965, 523.

[22] Williams (D. J.), Mulcahy (M. F. R.), Austral. J. Chem., 1966, 19, 2163.

[23] Kaufman (F.), Kelso (J. R.), J. Chem. Phys., 1967, 46, 4541.

[24] Francis (P. D.), Brit. J. Appl. Phys., 1969, 2, 1717.

[25] Slanger (T. G.), Black (G.), J. Chem. Phys., 1970, 53, $1,3717$.

[26] Stuhl (F.), Niki (H.), J. Chem. Phys., 1971, 55, 3943.

[27] Bauer (E.), J. Chem. Phys., 1960, 33, 1202. 